\title{
Spectroscopy of vanadium (III) doped gallium lanthanum sulphide chalcogenide glass
}

\author{
M. Hughes, ${ }^{a)}$ H. Rutt, and D. Hewak \\ Optoelectronics Research Centre, University of Southampton, Southampton SO17 1BJ, United Kingdom \\ R. J. Curry \\ Advanced Technology Institute, School of Electronics and Physical Sciences, University of Surrey, \\ Guildford GU2 7XH, United Kingdom
}

(Received 30 August 2006; accepted 14 December 2006; published online 17 January 2007)

\begin{abstract}
Vanadium doped gallium lanthanum sulphide glass (V:GLS) displays three absorption bands at 580, 730, and $1155 \mathrm{~nm}$ identified by photoluminescence excitation measurements. Broad photoluminescence, with a full width at half maximum of $\sim 500 \mathrm{~nm}$, is observed peaking at $1500 \mathrm{~nm}$ when exciting at 514, 808, and $1064 \mathrm{~nm}$. The fluorescence lifetime and quantum efficiency at $300 \mathrm{~K}$ were measured to be $33.4 \mu$ s and $4 \%$, respectively. From the available spectroscopic data, the authors propose the vanadium ions' valence to be $3+$ and be in tetrahedral coordination. The results indicate a potential for the development of a laser or optical amplifier based on V:GLS.
\end{abstract}

(C) 2007 American Institute of Physics. [DOI: 10.1063/1.2432280]

Chalcogenide glasses have low phonon energies due to the relatively large atomic mass of their constituent atoms. In particular, gallium lanthanum sulphide (GLS) glass has a maximum phonon energy of $425 \mathrm{~cm}^{-1}$, which gives it excellent infrared transmission up to $9 \mu \mathrm{m} .{ }^{1}$ This low maximum phonon energy enables emission from many transitions within active ion dopants, such as transition metals, which are weakly or not at all observed in other glasses (e.g., silica) due to their high phonon energies. In this letter we present spectroscopic data for vanadium doped GLS (V:GLS) glass. We assign a $3+$ oxidation state to the vanadium ion and energy levels to the observed transitions by comparisons to previous work on the spectroscopic analysis of $\mathrm{V}^{3+}$ in other hosts.

Samples of V:GLS were prepared by mixing $65 \%$ gallium sulphide $\left(\mathrm{Ga}_{x} \mathrm{~S}_{y}\right), 29.95 \%$ lanthanum sulphide $\left(\mathrm{La}_{2} \mathrm{~S}_{3}\right)$, 5\% lanthanum oxide $\left(\mathrm{La}_{2} \mathrm{O}_{3}\right)$, and $0.05 \%$ vanadium sulphide $\left(\mathrm{V}_{2} \mathrm{~S}_{3}\right)(\mathrm{mol} \%)$ in a dry-nitrogen purged glovebox. Gallium and lanthanum sulphides were synthesized in-house from gallium metal ( $9 N$ purity) and lanthanum fluoride ( $5 N$ purity) precursors in a flowing $\mathrm{H}_{2} \mathrm{~S}$ gas system. Before sulphurization, lanthanum fluoride was purified and dehydrated in a dry-argon purged furnace at $1250{ }^{\circ} \mathrm{C}$ for $36 \mathrm{~h}$ to reduce $\mathrm{OH}^{-}$ and transition metal impurities. The lanthanum oxide and vanadium sulphide were purchased commercially and used without further purification. The glass was melted in a dryargon purged furnace at $1150{ }^{\circ} \mathrm{C}$ for $24 \mathrm{~h}$ before being quenched and annealed at $400{ }^{\circ} \mathrm{C}$ for $12 \mathrm{~h}$.

Absorption spectra were taken on a Varian Cary 500 spectrophotometer over a range of $175-3300 \mathrm{~nm}$ with a resolution of $\pm 0.1 \mathrm{~nm}$. Samples were cut and polished into 5 and $0.5 \mathrm{~mm}$ thick slabs, which allowed reflection corrected absorption coefficient spectra to be calculated using.

${ }^{a)}$ Electronic mail: mah@orc.soton.ac.uk

$$
\alpha_{\mathrm{rc}}(\lambda)=\frac{A_{l_{1}}(\lambda)-A_{l_{2}}(\lambda)}{l_{1}-l_{2}},
$$

where $\alpha_{\mathrm{rc}}(\lambda)$ is the reflection corrected absorption coefficient spectrum and $A_{l_{i}}(\lambda)$ is the absorbance spectra of a sample of thickness $l_{i}$. Photoluminescence (PL) spectra were obtained by dispersing the fluorescence generated by laser sources at 514, 808, and $1064 \mathrm{~nm}$ in a Bentham TMc300 monochromator and detecting with a liquid nitrogen cooled InSb or InGaAs detector coupled with standard phase sensitive detection. All spectra were corrected for the system response. To obtain photoluminescence excitation (PLE) spectra, a $1400 \mathrm{~nm}$ long pass filter was placed in front of an InGaAs detector to give an effective detection range of $1400-1700 \mathrm{~nm}$. The excitation source used was a $250 \mathrm{~W}$ quartz halogen white light source passed through a monochromator with a $5 \mathrm{~nm}$ bandwidth. The PLE spectra were corrected for the varying intensity of exciting light due to varying grating response and spectral output of the white light source by characterizing the output of each grating with wavelength calibrated Newport 818-SL and 818-IG detectors and a Newport 1830-c optical power meter.

Fluorescence lifetime measurements were obtained using a $1064 \mathrm{~nm}$ Nd:YAG (yttrium aluminum garnet) laser modulated using a Gooch and Housego $80 \mathrm{MHz}$ acoustooptic modulator, and the fluorescence was detected with a New Focus $2053 \mathrm{InGaS}$ detector. The sample was placed in a Leybold AG helium gas closed cycle cryostat for cryogenic measurements.

Figure 1 shows the absorption spectra of undoped and $0.1 \%$ (molar) $\mathrm{V}^{3+}$ doped GLS. The undoped absorption spectrum is typical of GLS, showing a strong electronic absorption edge at $\sim 500 \mathrm{~nm}$. Within the absorption spectrum of the $0.1 \%$ V:GLS sample, a broad absorption centered at $\sim 1100 \mathrm{~nm}$ and a shoulder at $\sim 750 \mathrm{~nm}$ can be identified. The redshift of the absorption edge in the doped glass indicates that a third vanadium absorption band lies around $500 \mathrm{~nm}$. There is also evidence of a weak shoulder at $\sim 1000 \mathrm{~nm}$, which is attributed to a spin forbidden transition. No further 


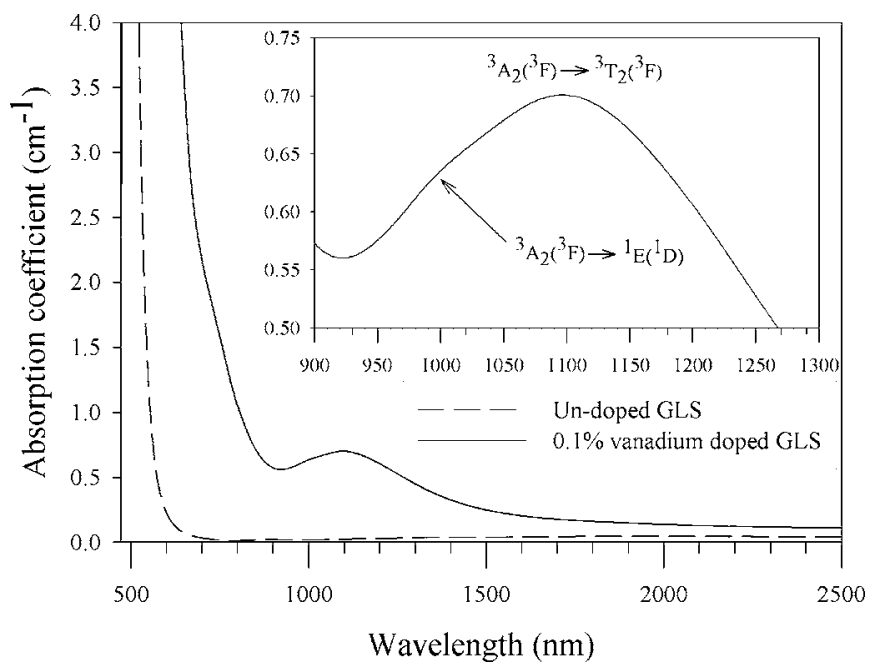

FIG. 1. Reflection corrected absorption spectra of $0.1 \%$ vanadium doped GLS and undoped GLS. Inset shows the peak at $1100 \mathrm{~nm}$ in detail.

absorption peaks were observed at wavelengths of up to $9 \mu \mathrm{m}$.

The PLE spectrum in Fig. 2 shows three broad Gaussian peaks located at 580, 730, and $1155 \mathrm{~nm}$, which clarifies the identification of the two high energy absorption bands in Fig. 1. The slight redshift of the strongest absorption band observed in the absorption and PLE spectra (centered at 1100 and $1155 \mathrm{~nm}$, respectively) can be reconciled by considering the overlap between the absorption and emission bands. When exciting at wavelengths longer than $1100 \mathrm{~nm}$, a greater proportion of ions sited in a lower crystal field (and hence fluorescing at longer wavelengths) will be excited. This will shift the PLE peak to longer wavelengths as there would be less reabsorption of fluorescence.

Figure 2 shows the room temperature PL spectra taken using laser excitation sources at 514, 808, and $1064 \mathrm{~nm}$, which roughly equates to exciting into each of the three absorption bands identified in Fig. 1 and 2. The PL spectra taken at each excitation wavelength show similar characteristic spectra peaking at $\sim 1500 \mathrm{~nm}$ with a full width at half maximum (FWHM) of $\sim 500 \mathrm{~nm}$. This indicates that the three absorption bands belong to the same oxidation state,

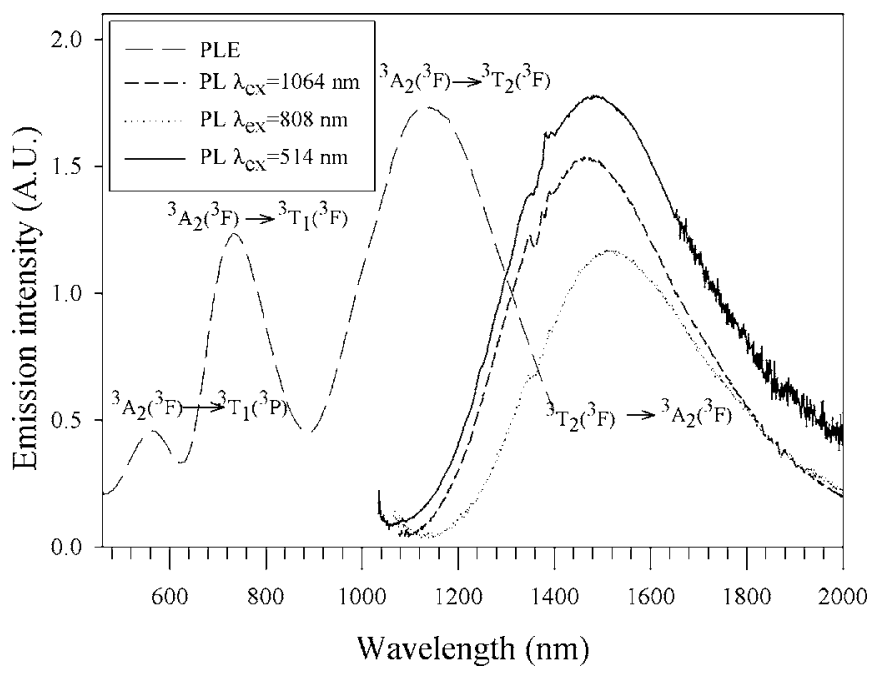

FIG. 2. Excitation and photoluminescence spectra of $0.01 \%$ vanadium

doped GLS.
Downloaded 30 Mar 2009 to 131.227.178.132. Redistribution subject to AlP license or copyright; see http://apl.aip.org/apl/copyright.jsp

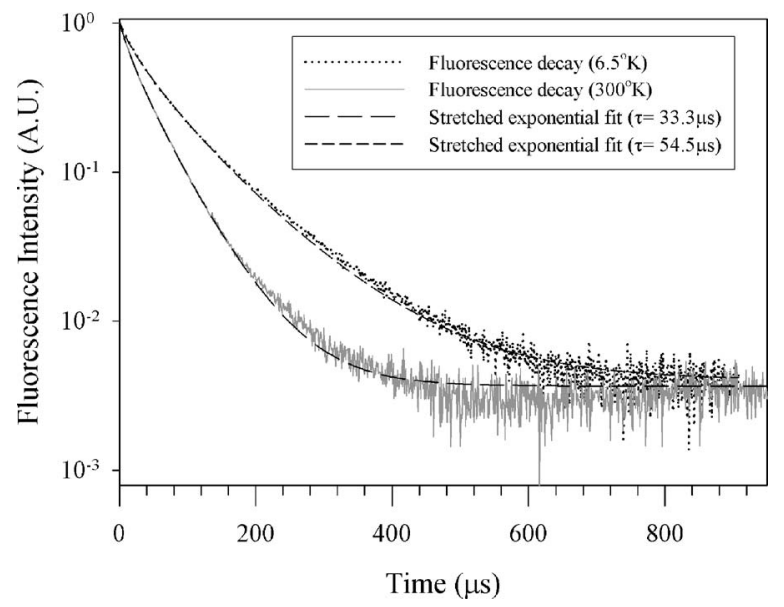

FIG. 3. Fluorescence decay of vanadium doped GLS at 6.5 and $300 \mathrm{~K}$ fitted with stretched exponentials.

rather than two or more oxidation states which is commonly observed in transition metal doped glasses ${ }^{2-4}$ and crystals. The broadness of the PL spectra indicates that the vanadium ion is in a low crystal field site. A result of this is that the ${ }^{3} T_{2}\left({ }^{3} F\right)$ level, which is strongly dependent on crystal field strength, is the lowest energy level. Conversely, in a strong crystal field site the ${ }^{1} E\left({ }^{1} D\right)$ level, which is almost independent of crystal field strength, is the lowest energy level. In this case, characteristic narrow $R$-line emission should be observed as in $\mathrm{V}^{3+}$ doped phosphate glass ${ }^{6}$ and $\mathrm{V}^{3+}$ doped corundum. ${ }^{7}$

The optical properties of V:GLS are similar to that of tetrahedrally coordinated $\mathrm{V}^{3+}$ in other hosts. Tetrahedral $\mathrm{V}^{3+}$ in YAG has three absorption bands centered at 600, 800, and $1320 \mathrm{~nm}$, which are attributed to spin allowed transitions from the ${ }^{3} A_{2}\left({ }^{3} F\right)$ ground state to the ${ }^{3} T_{1}\left({ }^{3} P\right),{ }^{3} T_{1}\left({ }^{3} F\right)$, and ${ }^{3} T_{2}\left({ }^{3} F\right)$ levels, respectively; a weak and narrow absorption at $1140 \mathrm{~nm}$ is attributed to the spin forbidden transition ${ }^{3} A_{2}\left({ }^{3} F\right)$ to ${ }^{1} E\left({ }^{3} D\right){ }^{5,8,9}$ Likewise, tetrahedral $\mathrm{V}^{3+}$ in $\mathrm{LiAlO}_{2}, \mathrm{LiGaO}_{2}$, and $\mathrm{SrAl}_{2} \mathrm{O}_{4}$ has three absorption bands centered $\sim 550$, 850 , and $1350 \mathrm{~nm}$, which are attributed to transitions from ${ }^{3} A_{2}\left({ }^{3} F\right)$ to ${ }^{3} T_{1}\left({ }^{3} P\right),{ }^{3} T_{1}\left({ }^{3} F\right)$, and ${ }^{3} T_{2}\left({ }^{3} F\right)$ levels, respectively. ${ }^{10}$ Optical transitions associated with octahedral $\mathrm{V}^{3+}$ tend to occur at higher energies than tetrahedral $\mathrm{V}^{3+}$. For example the ${ }^{3} T_{1}\left({ }^{3} F\right)$ to ${ }^{3} T_{2}\left({ }^{3} F\right)$ and ${ }^{3} T_{1}\left({ }^{3} P\right)$ transitions of octahedral $\mathrm{V}^{3+}$ :YAG occur at 600 and $425 \mathrm{~nm}$, respectively, ${ }^{5,8,11}$ at 707 and $440 \mathrm{~nm}$ in zirconium fluoride glass $^{12}$ and at 724 and $459 \mathrm{~nm}$ in phosphate glass. ${ }^{6}$

Dopant ions in glasses are generally expected to substitute for network modifier cations. ${ }^{13}$ The main network modifier in GLS is $\mathrm{La}^{3+} .{ }^{14}$ Using the approximate matrix elements of the Tanabe-Sugano model ${ }^{15}$ for a $3 d^{2}$ electronic configuration in octahedral coordination along with the absorption peaks at 1100 and $730 \mathrm{~nm}$, a crystal field strength $(D q / b)$ of 2.8 and a Racah $c$ parameter of $1570 \mathrm{~cm}^{-1}$ were calculated. This would mean that the $\mathrm{V}^{3+}$ ion is in a high field site, which is inconsistent with the FWHM and lifetime of the PL. The same calculation for a $3 d^{2}$ tetrahedral configuration gives $D q / b=1.6$ and $c=2460 \mathrm{~cm}^{-1}$, which mean a low field site, consistent with the FWHM and lifetime of the PL and the spin forbidden absorption at $1000 \mathrm{~nm}$. We therefore propose that $\mathrm{V}^{3+}$ is tetrahedrally coordinated, and attribute absorption peaks at 580,730, and $1100 \mathrm{~nm}$ to transitions from the ${ }^{3} A_{2}\left({ }^{3} F\right)$ ground state to the ${ }^{3} T_{1}\left({ }^{3} P\right),{ }^{3} T_{1}\left({ }^{3} F\right)$, and ${ }^{3} T_{2}\left({ }^{3} F\right)$ 
TABLE I. Overview of the spectroscopic parameters for various laser materials compared to V:GLS.

\begin{tabular}{lccccc}
\hline \hline \multicolumn{1}{c}{ Ion:Host } & $\tau(\mu \mathrm{s})$ & $\begin{array}{c}\eta_{\mathrm{QE}} \\
(\%)\end{array}$ & $\begin{array}{c}\sigma_{\mathrm{em}} \\
\left(10^{-2} \mathrm{~cm}^{2}\right)\end{array}$ & $\begin{array}{c}\sigma_{\mathrm{em}} \tau \\
\left(10^{-24} \mathrm{~s} \mathrm{~cm}^{2}\right)\end{array}$ & Ref. \\
\hline & & & & & This \\
$\mathrm{V}^{3+}: \mathrm{GLS}$ & 33 & 4 & 0.03 & 0.1 & work \\
$\mathrm{Cr}^{4+}: \mathrm{Y}_{2} \mathrm{SiO}_{5}$ & 1.3 & $<10$ & 2.0 & 0.26 & 24 \\
$\mathrm{Cr}^{4+}: \mathrm{YAl}_{5} \mathrm{O}_{12}$ & 4.1 & 22 & 3.3 & 1.35 & 25 \\
$\mathrm{~V}^{2+}: \mathrm{MgF}_{2}$ & 40 & & 0.08 & 0.32 & 26 \\
$\mathrm{Ti}^{3+}: \mathrm{Al}_{2} \mathrm{O}_{3}$ & 3.1 & 100 & 4.5 & 1.40 & 27 \\
\hline
\end{tabular}

levels; the weak shoulder at $1000 \mathrm{~nm}$ to the ${ }^{3} A_{2}\left({ }^{3} F\right)$ to ${ }^{1} E\left({ }^{1} D\right)$ transition; and the PL peaking at $1500 \mathrm{~nm}$ to the ${ }^{3} T_{2}\left({ }^{3} F\right)$ to ${ }^{3} A_{2}\left({ }^{3} F\right)$ transition. It is also noted that there is no significant change in absorption, photoluminescence, and lifetime of $\mathrm{V}^{3+}$ in the host gallium lanthanum oxy-sulphide, which contains $\sim 15 \%$ (molar) oxygen.

The fluorescence decay profiles were found to be nonexponential, but could be accurately described using a stretched exponential function: $I(t)=I_{0} \exp \left[-(t / \tau)^{p}\right]$. where $I_{0}$ is the initial fluorescence intensity, $\tau$ is the fluorescence lifetime, and $p$ is the stretch factor. The stretched exponential function is widely known to fit many different relaxation processes including charge relaxation, magnetic susceptibility relaxation, and fluorescence decay in many amorphous materials ${ }^{16,17}$ and crystalline solids. ${ }^{18}$ Stretched exponential decay behavior can be explained by the random network structure of glasses. ${ }^{19}$

Using this method to fit the fluorescence decay profiles shown in Fig. 3, lifetimes of 54.5 and $33.3 \mu$ s are obtained at 6.5 and $300 \mathrm{~K}$, respectively. This increase in lifetime with reduction in temperature is attributed to the depopulation of higher phonon levels at lower temperatures which reduces the probability of nonradiative decay. For comparison, the $300 \mathrm{~K}$ lifetimes for $\mathrm{V}^{3+}$ doped YAG, $\mathrm{LiAlO}_{2}$, and $\mathrm{LiGaO}_{2}$ are $22 \mathrm{~ns},{ }^{5} 0.5 \mu \mathrm{s},{ }^{20}$ and $11 \mu \mathrm{s},{ }^{20}$ respectively.

Quantum efficiency (QE) measurements were obtained by placing the sample in an integrating sphere and taking spectra of the fluorescence and of the $1064 \mathrm{~nm}$ exciting laser line, with and without the sample. A "photons out/photons in" method similar to that described elsewhere was used to calculate the QE. ${ }^{21,22}$ The photons out were calculated from the area under the fluorescence spectra, and the photons in were calculated from the difference in the area of the laser line spectra with and without the sample. The QE of $0.1 \%$ $\mathrm{V}$ :GLS was calculated to be $4.2 \%$.

The formula of McCumber ${ }^{23}$ in Eq. 3 is used to calculate the peak emission cross section, where $\lambda_{0}$ is the peak

$$
\sigma_{\mathrm{em}}=\sqrt{\frac{\ln 2}{\pi}} \frac{A}{4 \pi c n^{2}} \frac{\lambda_{0}^{4}}{\Delta \lambda}
$$

the fluorescence wavelength, $\Delta \lambda$ is the FWHM, $n$ is the refractive index, $c$ is the speed of light, and $A$ is the Einstein coefficient calculated from $\mathrm{QE} / \tau=1260 \mathrm{~s}^{-1}$. The calculation for V:GLS is given in Table I. To date there has been no demonstration of lasing from $\mathrm{V}^{3+}$, in part due to the small radiative lifetimes mentioned above. Table I compares the spectroscopic parameters from this work with those of other relevant laser materials doped with $\mathrm{Cr}^{4+}$ which is isoelectric to $\mathrm{V}^{3+}, \mathrm{V}^{2+}$, and the commercially successful Ti:sapphire. Comparisons indicate that the lifetime of V:GLS is comparable to or better than existing doped laser hosts. Though the $\mathrm{QE}$ and emission cross section do not compare favorably, the ability to form optical fibers from this material may overcome potential heat dissipation problems caused by the low QE (due to the large surface area to volume ratio of optical fibers). Additionally, the high pump beam confinement that can also be achieved in a fiber could compensate for the low emission cross section.

In summary, we have demonstrated strong, broad, room temperature emission peaking at $\sim 1500 \mathrm{~nm}$ from $\mathrm{V}^{3+}$ doped GLS glass when excited by laser sources into each of the three absorption bands at 580, 730, and $1100 \mathrm{~nm}$. The room temperature emission lifetime was measured to be $33.3 \mu$ s. We note that this system may have a potential for developing a low-cost V:GLS tunable laser or optical amplifier, with an optical fiber waveguiding structure being the best possibility for achieving this.

${ }^{1}$ D. J. Brady, T. Schweizer, J. Wang, and D. W. Hewak, J. Non-Cryst. Solids 242, 92 (1998)

${ }^{2}$ R. K. Brow, J. Am. Ceram. Soc. 70, 129 (1987).

${ }^{3}$ W. D. Johnstone, J. Am. Ceram. Soc. 48, 608 (1965).

${ }^{4}$ M. A. Noginov, J. Appl. Phys. 91, 569 (2002).

${ }^{5}$ A. M. Malyarevich, Appl. Phys. B: Lasers Opt. 67, 555 (1998).

${ }^{6}$ A. G. Khudoleev and N. M. Bokin, J. Appl. Spectrosc. 14, 75 (1971).

${ }^{7}$ Z. Goldschmidt, W. Low, and M. Foguel, Phys. Lett. 19, 17 (1965).

${ }^{8}$ K. V. Yumashev, N. V. Kuleshov, and A. M. Malyarevich, J. Appl. Phys. 80, 4782 (1996)

${ }^{9}$ V. P. Mikhailoz and N. V. Kuleshove, Opt. Mater. (Amsterdam, Neth.) 2 , 267 (1993).

${ }^{10}$ S. Kuck and P. Jander, Chem. Phys. Lett. 300, 189 (1999).

${ }^{11}$ M. J. Weber and L. A. Riseberg, J. Chem. Phys. 55, 2032 (1971).

${ }^{12}$ Y. Ohishi, S. Mitachi, T. Kanamori, and T. Manabe, Phys. Chem. Glasses 24, 135 (1983).

${ }^{13}$ B. Henderson and G. F. Imbusch, Optical Spectroscopy of Inorganic Solids (Oxford, New York, 1989), Chap. 9, p. 442.

${ }^{14}$ S. Benazeth, M. H. Tuilier, A. M. Loireau-Lozac'h, H. Dexpert, P. Lagarde, and J. Flahaut, J. Non-Cryst. Solids 110, 89 (1989).

${ }^{15} \mathrm{~S}$. Sugano, Y. Tanabe, and H. Kamimura, Multiplets of Transition-Metal Ions in Crystals (Academic, New York, 1970), Appendix IV, p. 295.

${ }^{16}$ J. C. Phillips, Rep. Prog. Phys. 59, 1133 (1996).

${ }^{17}$ H. Sillescu, J. Non-Cryst. Solids 243, 81 (1998).

${ }^{18}$ R. Chen, J. Non-Cryst. Solids 102-103, 510 (2003).

${ }^{19}$ R. A. L. Vallée, M. Cotlet, J. Hofkens, F. C. De Schryver, and K. Mullen, Macromolecules 36, 7752 (2003).

${ }^{20}$ S. Kück, Appl. Phys. B: Lasers Opt. 72, 515 (2001).

${ }^{21}$ C. E. Finlayson, A. Amezcua, P. J. Sazio, P. S. Walker, M. C. Grossel, R. J. Curry, D. C. Smith, and J. J. Baumberg, J. Mod. Opt. 52, 955 (2005).

${ }^{22}$ N. C. Greenham, Chem. Phys. Lett. 241, 89 (1995).

${ }^{23}$ D. E. McCumber, Phys. Rev. 136, 954 (1964).

${ }^{24}$ C. Deka, M. Bass, B. H. T. Chai, and Y. Shimony, J. Opt. Soc. Am. B 10, 1499 (1993).

${ }^{25}$ S. Kuck, K. Peterman, U. Pohlmann, and G. Huber, Phys. Rev. B 51, 323 (1995).

${ }^{26}$ R. Moncorge and T. Benyattou, Phys. Rev. B 37, 9177 (1988).

${ }^{27}$ P. Albers, E. Stark, and G. Huber, J. Opt. Soc. Am. B 3, 134 (1986). 